\title{
On conformal capacity and Teichmüller's modulus problem in space
}

\author{
Dimitrios Betsakos \\ Department of Mathematics, P.O. Box 4, \\ FIN-00014 University of Helsinki, Finland. \\ E-mail: betsakos@geom.helsinki.fi \\ FAX: 00358919123213.
}

March 22, 1999

FILE: te.tex

\begin{abstract}
We solve an extremal problem for the conformal capacity of certain space condensers. The extremal condenser is conformally equivalent to Teichmüller's ring. As an application, we give a dimension-free estimate for the minimal conformal capacity of the condensers with plates $E, F$ such that $a, b \in E, c, d \in F$, where $a, b, c, d$ are given points in $\overline{\mathbb{R}}^{n}$.
\end{abstract}

1991 Mathematics Subject Classification. 31B15, 30C85.

Key words and phrases. Condenser, conformal capacity, extremal length, Teichmüller's problem, polarization. 


\section{Introduction and results}

Notation: Let $\mathbb{R}^{n}, n \geq 2$, be the $n$-dimensional euclidean space, and $\overline{\mathbb{R}}^{n}$ its one-point compactification $\mathbb{R}^{n} \cup\{\infty\}$. The norm of a vector $x \in \mathbb{R}^{n}$ is written as $|x|=\left(x_{1}^{2}+\cdots+x_{n}^{2}\right)^{1 / 2}$, where $x_{1}, \ldots, x_{n}$ are the coordinates of $x$. By $e_{1}, e_{2}, \ldots, e_{n}$ we denote the standard unit vectors of $\mathbb{R}^{n}$. For $x, y \in \mathbb{R}^{n}$, we set $[x, y]=\{(1-t) x+t y: 0 \leq t \leq 1\}$, and for $x \neq O,[x, \infty]=\{t x: t \geq 1\} \cup\{\infty\}$, where $O$ is the origin. The $(n-1)$-dimensional sphere in $\mathbb{R}^{n}$ with center $a$ and radius $r$ will be denoted by $S^{n-1}(a, r)$.

A condenser in $\overline{\mathbb{R}}^{n}$ is a pair $(A, B)$, where $A, B$ are nonempty, disjoint, compact subsets of $\overline{\mathbb{R}}^{n}$ with $\infty \in A$. The complementary sets $A$ and $B$ are the plates of the condenser, and the open set $R=\overline{\mathbb{R}}^{n} \backslash(A \cup B)$ is the field of the condenser. If both $A$ and $B$ are connected, then the condenser $(A, B)$ is called a ring. The conformal capacity of $(A, B)$ is defined as

$$
\operatorname{cap}(A, B)=\inf _{u} \int_{R}|\nabla u|^{n} d x
$$

where the infimum is taken over all nonnegative $C^{\infty}(R \cup B)$ functions with compact support in $R \cup B$ such that $u(x) \geq 1$, for $x \in B$ (cf. [AVV] p.165).

Inequalities for the conformal capacity of condensers play an important role in quasiconformal maps (see, e.g. [G1], [V1], [AVV]). In this paper we study rings whose plates are rectilinear segments or half-lines. For such rings F.W.Gehring (see [V1] Lemma 5.27) proved the following result which has many applications in the distortion theory of quasiconformal mappings (see [V1], [AVV]).

1.2. Theorem. Let $e \in S^{n-1}(O, 1)$ and $s>1$. Then

$$
\operatorname{cap}\left(\left[O, e_{1}\right],\left[s e_{1}, \infty\right]\right) \geq \operatorname{cap}\left([O, e],\left[s e_{1}, \infty\right]\right) .
$$
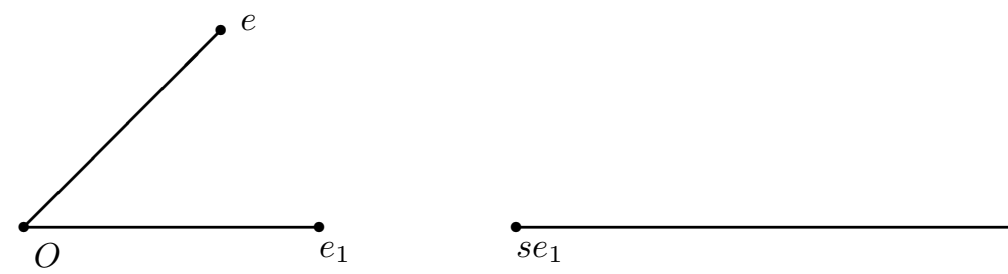

Figure 1: An illustration for Theorem 1.2 .

We shall prove the following stronger result.

1.4. Theorem. Let $0<r \leq s \leq t \leq \rho, \theta \in[0, \pi / 2]$ and $e=(\cos \theta) e_{1}+$ $(\sin \theta) e_{n}$. Consider the sets $E=[-r e, s e], E^{\#}=\left[-r e_{1}, s e_{1}\right], F=\left\{l e_{1}: l \geq\right.$ $t, l \leq-\rho\}$. Then

$$
\operatorname{cap}(E, F) \leq \operatorname{cap}\left(E^{\#}, F\right) .
$$



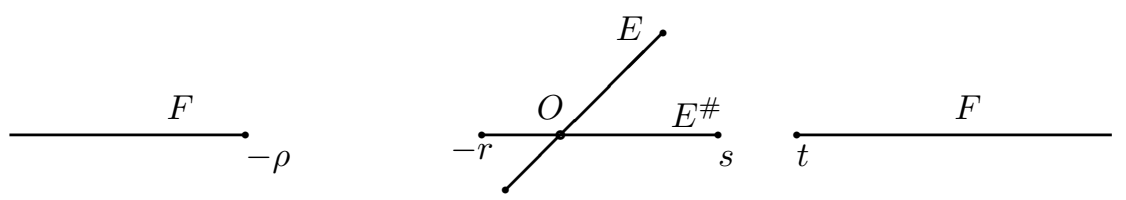

Figure 2: An illustration for Theorem 1.4.

The inequality (1.5) implies the "conjecture for chapter 8" in the Appendix $\mathrm{F}$ of the book $[\mathrm{AVV}]$ by G.D.Anderson, M.K.Vamanamurthy, and M.Vuorinen.

For $n=2$, an even stronger result can be easily proved: Let $f(\theta)$ denote the conformal capacity of the condenser $(E, F)$. Then $f$ is strictly decreasing for $\theta \in(0, \pi / 2)$. This can be proved by mapping the domain $\mathbb{R}^{2} \backslash E^{\#}$ conformally onto $\{z:|z|>1\}$ and then using simple geometric arguments involving ellipses. Such a monotonicity result probably holds in every dimension.

The proof of Theorem 1.4 is given in Section 3.

In Section 4 we apply Theorem 1.4 to Teichmüller's modulus problem in space. For $x \in \mathbb{R}^{n} \backslash\left\{O, e_{1}\right\}$, let

$$
p(x)=\inf _{E, F} \operatorname{cap}(E, F),
$$

where the infimum is taken over all pairs of continua $E$ and $F$ with $O, e_{1} \in E$ and $x, \infty \in F$. For $n=2$, the problem of determining the value of $p(x)$ for all $x$ and finding the corresponding extremal continua was posed by O.Teichmüller $[\mathrm{Te}]$. For further work in the two-dimensional case we refer to $[\mathrm{Ku}]$ ch.5 and [SV], and to the references therein. For $n \geq 3$, the problem was studied in [V1] ch.8, [V2] and [AVV] ch.15.

Here we are interested in upper bounds of the form

$$
p(x) \leq c \tau_{n}\left(\min \left\{|x|,\left|x-e_{1}\right|\right\}\right),
$$

where $c$ is a constant ant $\tau_{n}(r)$ is the conformal capacity of Teichmüller's ring $\left(\left[-e_{1}, O\right],\left[r e_{1}, \infty\right]\right)$. By symmetry, we may assume that $\left|x-e_{1}\right| \leq|x|$. Vuorinen [V2] proved that

$$
p(x) \leq \tau_{n}\left(\frac{|x|+\left|x-e_{1}\right|-1}{2}\right), x \in \mathbb{R}^{n} \backslash\left[O, e_{1}\right] .
$$

This implies ([AVV] p.304) that

$$
p(x) \leq \sqrt{2} \tau_{n}\left(\left|x-e_{1}\right|\right), \quad|x|>1 .
$$

For general $x \in \mathbb{R}^{n} \backslash\left\{e_{1}\right\},\left|x-e_{1}\right| \leq|x|$, [AVV] gives the bound

$$
p(x) \leq 2^{n} \sqrt{3} \tau_{n}\left(\left|x-e_{1}\right|\right) .
$$


Vuorinen posed the problem whether the number $2^{n} \sqrt{3}$ in (1.8) can be replaced by a number independent of $n$, (see [V2] 3.11 open problem (2) and [AVV] p.312). We answer this question in the affirmative:

1.9. Theorem. For $x \in \mathbb{R}^{n} \backslash\left\{e_{1}\right\},\left|x-e_{1}\right| \leq|x|$,

$$
p(x) \leq 4 \tau_{n}\left(\left|x-e_{1}\right|\right)
$$

The proof of Theorem 1.9 is based on Theorem 1.4 and on V.N.Dubinin's polarization inequalities for condensers [Du]. In Section 2 we review some known results on conformal capacity and polarization that are necessary for the proofs of the theorems.

\section{Background}

\subsection{Conformal capacity and extremal length}

Let $\Gamma$ be a family of curves in $\overline{\mathbb{R}}^{n}$. By $\mathcal{F}_{\Gamma}$ we denote the family of all nonnegative Borel functions $\rho: \mathbb{R}^{n} \longrightarrow \mathbb{R} \cup\{\infty\}$ such that

$$
\int_{\gamma} \rho d s \geq 1,
$$

for every locally rectifiable curve $\gamma \in \Gamma$. The modulus $M(\Gamma)$ and the extremal length $\lambda(\Gamma)$ are defined by

$$
\begin{aligned}
& M(\Gamma)=\inf _{\rho} \int_{\mathbb{R}^{n}} \rho^{n} d m, \\
& \lambda(\Gamma)=M(\Gamma)^{1 /(1-n)},
\end{aligned}
$$

where the infimum is taken over all $\rho \in \mathcal{F}_{\Gamma}$ and $m$ is the $n$-dimensional Lebesgue measure.

If $E, F \subset \overline{\mathbb{R}}^{n}$ and $\Gamma$ is the family of all curves joining $E$ and $F$ then we write $M(E, F)=M(\Gamma)$ and $\lambda(E, F)=\lambda(\Gamma)$. For the properties of modulus and extremal length we refer to $[\mathrm{AVV}] \mathrm{ch} .8$ and the references therein. Here we mention only two main properties:

2.5. Theorem. Let $f$ map the domain $D \subset \overline{\mathbb{R}}^{n}$ conformally onto a domain $D^{\prime} \subset \overline{\mathbb{R}}^{n}$. Then $M(f(\Gamma))=M(\Gamma)$, for each curve family $\Gamma$ in $D^{\prime}$.

A theorem due to F.W.Gehring [G2] and W.P.Ziemer [Zi] states that the conformal capacity of a condenser is equal to the modulus of the family of curves joining its plates:

2.6. Theorem. If $(E, F)$ is a condenser in $\overline{\mathbb{R}}^{n}$, then

$$
\operatorname{cap}(E, F)=M(E, F) \text {. }
$$




\subsection{Teichmüller's ring}

The plates of Teichmüller's ring are $\left[-e_{1}, O\right]$ and $\left[r e_{1}, \infty\right]$, where $r>0$. Its capacity is denoted by $\tau_{n}(r)$. For $n=2, \tau_{n}$ has an explicit expression in terms of elliptic integrals (see [AVV] ch.8). For $n \geq 3$, there are no known expressions and so we rely on qualitative properties and inequalities: $\tau_{n}$ is a strictly decreasing function of $r$ and satisfies the following inequality ([AVV] Theorem 11.25)

$$
\tau_{n}(c r) \leq \frac{1}{\sqrt{c}} \tau_{n}(r), 0<c \leq 1, r>0 .
$$

The ring $\left(\left[-e_{1}, e_{1}\right],\left[-b e_{1}, \infty\right] \cup\left[b e_{1}, \infty\right]\right)$ can be mapped by a Mbius transformation onto Teichmüller's ring. Using this fact we easily find that (see [AVV] exercise $7.52(\mathrm{c}))$

$$
\operatorname{cap}\left(\left[-e_{1}, e_{1}\right],\left[-b e_{1}, \infty\right] \cup\left[b e_{1}, \infty\right]\right)=\tau_{n}(t),
$$

where $t=(b-1)^{2} /(4 b)$. 


\subsection{Polarization}

Polarization is a geometric transformation in $\overline{\mathbb{R}}^{n}$ discovered by V.Wolontis [Wo]. The behaviour of the conformal capacity of condensers under polarization was investigated by V.N.Dubinin [Du] who used it to solve problem 7.57 in $[\mathrm{BBH}]$.

For $x=\left(x_{1}, \ldots, x_{n}\right) \in \mathbb{R}^{n}$, let $x^{*}=\left(x_{1}, \ldots, x_{n-1},-x_{n}\right)$. Let also $\infty^{*}=$ $\infty$. If $A$ is a compact subset of $\overline{\mathbb{R}}^{n}$, let $A^{*}=\left\{x^{*}: x \in A\right\}, A^{+}=\{x \in A$ : $\left.x_{n} \geq 0\right\}, A^{-}=\left\{x \in A: x_{n} \leq 0\right\}$. The polarization $\mathbb{P} A$ of $A$ with respect to the oriented $(n-1)$-dimensional plane $\left\{x_{n}=0\right\}$ is defined by

$$
\mathbb{P} A=\left(A \cup A^{*}\right)^{+} \cup\left(A \cap A^{*}\right)^{-} .
$$

If $(A, B)$ is a condenser in $\overline{\mathbb{R}}^{n}$ the polarization $\mathbb{P}(A, B)$ of $(A, B)$ with respect to $\left\{x_{n}=0\right\}$ is the condenser

$$
\mathbb{P}(A, B)=\left(\mathbb{P} A,(\mathbb{P} B)^{*}\right) .
$$

Note that $\mathbb{P}(A, B)=\mathbb{P}\left(B^{*}, A^{*}\right)$ and therefore

$$
\text { cap } \mathbb{P}(A, B)=\operatorname{cap} \mathbb{P}(B, A) .
$$

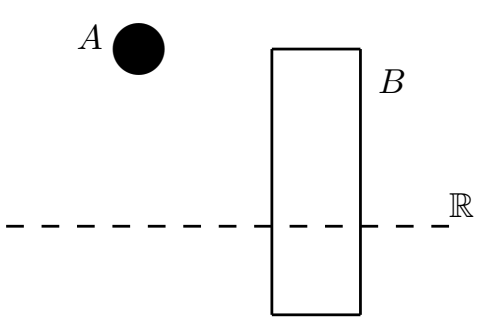

$A$

Figure 3: A condenser $(A, B)$ in $\mathbb{R}^{2}$ and its polarization $\left(\mathbb{P} A,(\mathbb{P} B)^{*}\right)$.

Now, let $H$ be an oriented $(n-1)$-dimensional plane or sphere in $\mathbb{R}^{n}$. Let $T$ be a Mbius transformation that maps $\left\{x_{n}=0\right\}$ (with its natural orientation) onto $H$ preserving their orientation. Then we define the polarization $\mathbb{P}_{H} A$ of a compact set $A \subset \overline{\mathbb{R}}^{n}$ by

$$
\mathbb{P}_{H} A=T \mathbb{P} T^{-1} A
$$

and similarly we define the polarization $\mathbb{P}_{H}(A, B)$ of the condenser $(A, B)$ with respect to $H$.

Dubinin $[\mathrm{Du}]$ proved the following theorem: 
2.15. Theorem. If $(A, B)$ is a condenser in $\mathbb{R}^{n}$ and $H$ is an oriented $(n-1)$-dimensional plane or sphere, then

$$
\operatorname{cap}(A, B) \geq \operatorname{cap} \mathbb{P}_{H}(A, B)
$$

2.17. Remark. Theorem 1.2 follows easily from Theorem 2.15.

\section{The inequality for conformal capacity}

In this section we prove Theorem 1.4. We start with three lemmas.

3.1. Lemma. Let $k, l, \epsilon$ be real numbers such that $k<l, k+l>0$ and $l+\epsilon<1$.Then

$$
\left.\lambda\left(\left[k e_{1}, l e_{1}\right], S^{n-1}(O, 1)\right) \geq \lambda\left[(k+\epsilon) e_{1},(l+\epsilon) e_{1}\right], S^{n-1}(O, 1)\right) .
$$

Proof. The inequality (3.2) follows at once by applying a polarization with respect to the $(n-1)$-dimensional plane which is perpendicular to the $e_{1^{-}}$ axis at the point $(k+l+\epsilon) e_{1} / 2$, and has orientation such that $\left[(k+\epsilon) e_{1},(l+\epsilon) e_{1}\right]$ is the polarization of $\left[k e_{1}, l e_{1}\right]$.

3.3. Lemma. Let $b \leq 0 \leq k \leq l$ with $l-b<1$. For $z \in \mathbb{C}$, define

$$
g(z)=\frac{z+b}{1+b z}
$$

Then

$$
\begin{gathered}
g(-b-l) \leq g(-b-k) \leq g(-b+k) \leq g(-b+l), \\
g(-b-k)-g(-b-l) \leq g(-b+l)-g(-b+k), \\
g(-b)-g(-b-k) \leq g(-b+k)-g(-b), \\
g(-b)-g(-b-l) \leq g(-b+l)-g(-b) .
\end{gathered}
$$

Proof. The function $g$ is a Mbius transformation that maps the unit disk onto itself with $g(0)=b, g(-b)=0, g(1)=1, g(-1)=-1$. Hence $g$ preserves the diameter $[-1,1]$ and its orientation. In particular (3.4) holds. The inequality (3.5) follows from the fact that $g(x)$ is a convex function on $[-1,1]\left(b \leq 0 \Rightarrow g^{\prime \prime} \geq 0\right)$. Finally (3.6) and (3.7) follow from the fact that $g$ preserves hyperbolic distances (or by a simple computation). 
3.8. Lemma. Let $a \leq 0<r \leq s<R, \theta \in[0, \pi / 2], e=(\cos \theta) e_{1}+$ $(\sin \theta) e_{n}$, and $E=[-r e, s e]$. Let $\gamma$ be the hyperbolic geodesic segment of $S=$ $S^{n-1}\left(a e_{1}, R\right)$ that lies in the $e_{1} e_{n}$-plane, is tangent to $E$ at the origin, and has end-points $A \in S^{n-1}(O, r) \cap\left\{x_{n} \leq 0\right\}$ and $B \in S^{n-1}(O, s) \cap\left\{x_{n} \geq 0\right\}$. Then

$$
\lambda(E, S) \geq \lambda(\gamma, S) .
$$

Proof. Let $j$ be the reflection with respect to $S^{n-1}(O, r)$. Then $j(E)$ and $j(\gamma)$ lie on two parallel straight lines $l_{R}$ and $l_{\gamma}$, respectively. Also, $j(S)$ is a sphere centered at $r^{2} e_{1} / a$ and such that

$$
j(S) \subset j\left(S^{n-1}(O, s)\right) \subset S^{n-1}(O, r) .
$$

Moreover, $l_{\gamma}$ is perpendicular to $j(S)$, while $l_{E}$ is perpendicular to $S^{n-1}(O, r)$ and to $j\left(S^{n-1}(O, s)\right)$. These geometric relations and a polarization $\mathbb{P}_{H}$ with respect to the $(n-1)$-dimensional plane $H$ which is perpendicular to the $e_{1} e_{n^{-}}$ plane and such that $\mathbb{P}_{H}\left(l_{\gamma}\right)=l_{R}$ show that (3.9) holds.

\subsection{Proof of Theorem 1.4}

By (2.4) and Theorem 2.6, it suffices to prove

$$
\lambda(E, F) \geq \lambda\left(E^{\#}, F\right) .
$$

Let $S=S^{n-1}\left(a e_{1}, R\right)$ be the sphere in $\mathbb{R}^{n}$ such that $F$ is the reflection of $E^{\#}$ in $S$. A direct calculation gives

$$
\begin{gathered}
a=\frac{t s-r \rho}{s+t+r+\rho}, \\
R=\sqrt{(r+a)(\rho+a)}=\sqrt{(t-a)(s-a)} .
\end{gathered}
$$

Case 1: $t s \leq r \rho$.

In this case $a \leq 0$, because of (3.12). By symmetry and subadditivity of extremal length (see $[\mathrm{AVV}]$ Lemmas 8.15 and 8.5(2)),

$$
\begin{gathered}
\lambda\left(E^{\#}, F\right)=\lambda\left(E^{\#}, S\right)+\lambda(S, F), \\
\lambda(E, F) \geq \lambda(E, S)+\lambda(S, F) .
\end{gathered}
$$

So, it suffices to prove

$$
\lambda(E, S) \geq \lambda\left(E^{\#}, S\right) .
$$

We identify the $e_{1} e_{n}$-plane with $\mathbb{C}$ and the $e_{1}$-axis with $\mathbb{R}$ (just in order to use complex notation). Let $\Gamma$ be the geodesic of $S$ that lies in $\mathbb{C}$ and is tangent 
to $E$ at the origin. Let $\gamma$ be the subarc of $\Gamma$ with end-points $A, B$ such that $A \in S^{n-1}(O, r) \cap\left\{x_{n} \leq 0\right\}$ and $B \in S^{n-1}(O, s) \cap\left\{x_{n} \geq 0\right\}$. By Lemma 3.8,

$$
\lambda(E, S) \geq \lambda(\gamma, S) .
$$

Consider the conformal mapping $F$ that maps $S$ onto $S_{o}:=S^{n-1}(O, 1)$ with $F(O)=O, F(\overline{\mathbb{C}})=\overline{\mathbb{C}}$, and $F(\overline{\mathbb{R}})=\overline{\mathbb{R}}$. Then the restriction of $F$ to $\mathbb{C}$ is given by

$$
F=T \circ T_{2} \circ T_{1},
$$

where $T_{1}(z)=z-a$ is a translation, $T_{2}(z)=z / R$ is a streching, and $T$ is the Möbius map given by

$$
T(z)=\frac{z+a / R}{1+z a / R} .
$$

Let $\delta:=F(\gamma)$. Then, by the conformal invariance of extremal length (Theorem $2.5)$,

$$
\lambda(\gamma, S)=\lambda\left(\delta, S_{o}\right) .
$$

Since $F$ preserves geodesics and $\delta$ passes through the center $O$ of $S_{o}$, we conclude that $\delta$ is a rectilinear segment. The smallest angle between $\delta$ and $\mathbb{R}$ is equal to $\theta$. We write $\delta=\delta(\theta)$. Let

$$
\hat{s}:=F(-s), \quad s^{\prime}:=F(s), \quad \hat{r}:=F(-r), \quad r^{\prime}:=F(r) .
$$

Then, applying Lemma 3.3 (with $b=a / R$ ) we obtain

$$
\hat{s} \leq \hat{r} \leq r^{\prime} \leq s^{\prime}, \quad \hat{r}-\hat{s} \leq s^{\prime}-r^{\prime}, \quad-\hat{r} \leq r^{\prime}, \quad-\hat{s} \leq s^{\prime} .
$$

These inequalities show that the centers $r_{1}, s_{1}$ of the circles $F\left(S^{n-1}(O, r)\right) \cap$ $\mathbb{C}$ and $F\left(S^{n-1}(O, s)\right) \cap \mathbb{C}$, respectively, satisfy the inequalities $0 \leq r_{1} \leq s_{1}$. Therefore $\delta(\theta)$ contains a chord of the circle $F\left(S^{n-1}(O, r)\right) \cap \mathbb{C}$ and is contained in a chord of the circle $F\left(S^{n-1}(O, s)\right) \cap \mathbb{C}$; and these chords are diameters only if $\theta=0$.

Let $\delta^{\prime}(\theta):=e^{-i \theta} \delta(\theta)$. Then $\delta^{\prime}(\theta)$ is a segment $\left[\sigma_{1}, \sigma_{2}\right] \subset \mathbb{R}$ centered at a point of the positive real axis and $\sigma_{2} \leq s^{\prime}$. Moreover, for the length $\left|\delta^{\prime}(\theta)\right|$ of $\delta^{\prime}(\theta)$ we have

$$
\left|\delta^{\prime}(\theta)\right|=|\delta(\theta)| \leq|\delta(0)|=\left|\delta^{\prime}(0)\right| .
$$

Because of Lemma 3.1 these geometric properties imply

$$
\lambda\left(\delta(\theta), S_{o}\right)=\lambda\left(\delta^{\prime}(\theta), S_{o}\right) \geq \lambda\left(\delta(0), S_{o}\right)=\lambda\left(E^{\#}, S\right) .
$$

The last equality comes from conformal invariance; note that

$$
F\left(E^{\#}\right)=F([-r, s])=\left[\hat{r}, s^{\prime}\right]=\delta(0) .
$$


Now, (3.17), (3.18), and (3.19) show that (3.16) holds and the theorem is proved in case 1.

Case 2: $t s>r \rho$.

In this case we apply a reflection in $S^{n-1}(O, 1)$. Let $\mathcal{R}$ denote this reflection. By conformal invariance, (3.11) is equivalent to

$$
\lambda(\mathcal{R}(E), \mathcal{R}(F)) \geq \lambda\left(\mathcal{R}\left(E^{\#}\right), \mathcal{R}(F)\right) .
$$

But

$$
\begin{aligned}
\mathcal{R}\left(E^{\#}\right) & =\left\{l e_{1}: l \leq r^{-1}, l \geq s^{-1}\right\} \\
\mathcal{R}(F) & =\left[-\rho^{-1} e_{1}, t^{-1} e_{1}\right] \\
\mathcal{R}(E) & =\left\{l e: l \geq r^{-1}, l \leq s^{-1}\right\} .
\end{aligned}
$$

Define

$$
\begin{aligned}
\tilde{E} & :=\left[-\rho^{-1} e, t^{-1} e\right], \\
\tilde{F} & :=\left\{l e_{1}: l \leq-r^{-1}, l \geq s^{-1}\right\}, \\
\tilde{E}^{\#} & :=\left[-\rho^{-1} e_{1},{ }^{-1} e_{1}\right] .
\end{aligned}
$$

A rotation shows that $(3.20)$ is equivalent to

$$
\lambda(\tilde{E}, \tilde{F}) \geq \lambda\left(\tilde{E}^{\#}, \tilde{F}\right) .
$$

Let

$$
\tilde{r}:=\rho^{-1}, \quad \tilde{s}:=t^{-1}, \quad \tilde{t}:=s^{-1}, \quad \tilde{\rho}:=r^{-1} .
$$

Then $\tilde{t} \tilde{s}<\tilde{r} \tilde{\rho}$, and hence (3.23) follows from Case 1 . Thus the theorem is now proved.

\section{Teichmüller's modulus problem}

This section contains the proof of Theorem 1.9. Let $x=\left(x_{1}, \ldots, x_{n}\right) \in \mathbb{R}^{n} \backslash$ $\left\{O, e_{1}\right\}$. To prove the theorem, we may assume that $x_{2}=\cdots=x_{n-1}=0$ and $\left|x-e_{1}\right| \leq|x| \leq 1$. Let $\Omega$ be the region in the $e_{1} e_{n}$-plane defined by these conditions. So we have to prove that

$$
p(x) \leq 4 \tau_{n}\left(\left|x-e_{1}\right|\right), x \in \Omega .
$$

By the definition of $p(x)$, if $E, F$ are curves in $\overline{\mathbb{R}}^{n}$ with $O, e_{1} \in E$ and $x, \infty \in F$ then $p(x) \leq M(E, F)$. Moreover, by the subadditivity of moduli ([AVV] Lemma 
$8.3(3))$, if $F=F_{1} \cup F_{2}$ then $M(E, F) \leq M\left(E, F_{1}\right)+M\left(E, F_{2}\right)$. This type of argument will be repeatedly used in the proof below.

We will prove four lemmas. Theorem 1.9 is an immediate consequence of Lemmas 4.2, 4.13 and 4.16 .

4.2. Lemma. Suppose that $p(x) \leq 4 \tau_{n}\left(\left|x-e_{1}\right|\right.$ for all $x \in\left[e_{1} / 2, e_{1}\right] \cup$ $\left[e_{1} / 2, e_{1} / 2+\sqrt{3} e_{n} / 2\right]$. Then

$$
p(x) \leq 4 \tau_{n}\left(\left|x-e_{1}\right|\right) \text { for all } x \in \Omega .
$$

Proof. Suppose first that $x \in \Omega$ and $\left|x-e_{1}\right| \leq 1 / 2$. By applying a polarization with respect to a suitable $(n-1)$-dimensional plane perpendicular to the $e_{1} e_{n}$-plane and passing from $e_{1}$, we deduce that $p(x) \leq p\left(x^{\prime}\right)$, where $x^{\prime}=\left(1-\left|x-e_{1}\right|\right) e_{1}$ is the circular projection of $x$ on $\left[e_{1} / 2, e_{1}\right]$. On the other hand, by the definition of $x^{\prime},\left|x-e_{1}\right|=\left|x^{\prime}-e_{1}\right|$.

Suppose next that $x \in \Omega$ and $\left|x-e_{1}\right|>1 / 2$. We use the same polarization argument as above, but in this case we circularly project $x$ on $\left[e_{1} / 2, e_{1} / 2+\right.$ $\left.\sqrt{3} e_{n} / 2\right]$.

4.3. Lemma. Let $E=\left[-e_{1}, e_{1}\right]$ and $F=\left[-e_{n}, \infty\right]$. Then

$$
\begin{gathered}
\operatorname{cap}(E, F) \leq \tau_{n}(1)+\tau_{n}(1 / 3), \\
\operatorname{cap}(E, F) \leq 2 \tau_{n}(1 / 2) .
\end{gathered}
$$

Proof. Because of Theorem 2.6, we can use the modulus $M(E, F)$ instead of $\operatorname{cap}(E, F)$. Let $F_{1}=\left[-e_{n},-3 e_{n}\right], F_{2}=\left[-3 e_{n}, \infty\right]$. Then

$$
M(E, F) \leq M\left(E, F_{1}\right)+M\left(E, F_{2}\right)
$$

Now, by Theorem 1.4,

$$
M\left(E, F_{2}\right) \leq \tau_{n}\left(\frac{3-1}{2}\right)=\tau_{n}(1) .
$$

To estimate $M\left(E, F_{1}\right)$ we apply a polarization with respect to the sphere $S^{n-1}\left(-3 e_{n} / 2,3 / 2\right)$ and get

$$
M\left(E, F_{1}\right) \leq M\left(E,\left[-3 e_{n}, \infty\right] \cup\left[3 e_{n}, \infty\right]\right) .
$$

But by Theorem 1.4 and (2.10),

$$
M\left(E,\left[-3 e_{n}, \infty\right] \cup\left[3 e_{n}, \infty\right]\right) \leq \tau_{n}\left(\frac{1}{3}\right)
$$

Hence (4.4) is proved.

To prove (4.5), we let $L_{1}=\left[-e_{n},-2 e_{n}\right]$ and $L_{2}=\left[-2 e_{n}, \infty\right]$. Then $L_{2}$ is the reflection of $L_{1}$ in the sphere $S^{n-1}\left(-e_{n}, 1\right)$. By a polarization with respect to that sphere, 


$$
M\left(E, L_{1}\right) \leq M\left(E, L_{2}\right) .
$$

Hence

$$
M(E, F) \leq M\left(E, L_{1}\right)+M\left(E, L_{2}\right) \leq 2 M\left(E, L_{2}\right) .
$$

Finally, by Theorem 1.4,

$$
M\left(E, L_{2}\right) \leq \tau_{n}\left(\frac{2-1}{2}\right)=\tau_{n}\left(\frac{1}{2}\right)
$$

and the lemma is proved.

4.13. Lemma. Let $x=e_{1} / 2+x_{n} e_{n} \in \Omega$. Then

$$
p(x) \leq 3.9 \tau_{n}\left(\left|x-e_{1}\right|\right) .
$$

Proof. We identify the $e_{1} e_{n}$-plane with $\mathbb{C}$ and the $e_{1}$-axis with $\mathbb{R}$. We consider the Mbius map $T$ on $\overline{\mathbb{R}}^{n}$ whose restriction to $\mathbb{C}$ has the form

$$
T(z)=\frac{1-(2 z-1) i}{2 z-1-i} .
$$

Then $T(0)=-1, T(1)=1, T(\infty)=-i, T(1 / 2-i / 2)=\infty$, and

$$
T(x)=T\left(e_{1} / 2+x_{n} e_{n}\right)=\frac{1+2 x_{n}}{1-2 x_{n}} i .
$$

Let $E=[-1,1], F_{1}=[-i,-3 i], F_{2}=[i, 3 i], F_{3}=[-3 i, \infty] \cup[3 i, \infty]$.

Case 1: $0 \leq x_{n} \leq 1 / 4$.

In this case $T(x) \in[i, 3 i]$. Hence,

$$
p(x) \leq M\left(E, F_{1}\right)+M\left(E, F_{2}\right)+M\left(E, F_{3}\right) .
$$

As in the proof of Lemma 4.3,

$$
M\left(E, F_{1}\right)=M\left(E, F_{2}\right) \leq M\left(E, F_{3}\right) \leq \tau_{n}(1 / 3) .
$$

Therefore, by (4.14) and (2.9),

$$
\begin{gathered}
p(x) \leq 3 \tau_{n}\left(\frac{1}{3}\right)=3 \tau_{n}\left(\frac{1}{3} \frac{4}{\sqrt{5}} \frac{\sqrt{5}}{4}\right) \leq 3 \sqrt{\frac{3 \sqrt{5}}{4}} \tau_{n}\left(\frac{\sqrt{5}}{4}\right) \leq \\
\leq 3.9 \tau_{n}\left(\frac{\sqrt{5}}{4}\right) \leq 3.9 \tau_{n}\left(\left|x-e_{1}\right|\right) .
\end{gathered}
$$

The last inequality holds because if $x_{n} \in[0,1 / 4]$ then $\left|x-e_{1}\right|=\left(x_{n}^{2}+(1 / 2)^{2}\right)^{1 / 2} \leq$ $\sqrt{5} / 4$. 
Case 2: $1 / 4 \leq x_{n}<1 / 2$.

In this case $T(x) \in[3 i, \infty]$. Hence, by $(2.9)$,

$$
\begin{gathered}
p(x) \leq M\left(E, F_{1}\right)+M\left(E, F_{3}\right) \leq \tau_{n}(1 / 3)+\tau_{n}(1 / 3) \leq 2 \sqrt{3} \tau_{n}(1) \leq \\
\leq 3.5 \tau_{n}(1) \leq 3.5 \tau_{n}\left(\left|x-e_{1}\right|\right) .
\end{gathered}
$$

The last inequality comes from the fact $\left|x-e_{1}\right| \leq 1$, for $x \in \Omega$.

Case 3: $1 / 2 \leq x_{n} \leq \sqrt{3} / 2$.

In this case $T(x) \in[-i, \infty]$. Hence

$$
p(x) \leq M\left(E, F_{1}\right)+M\left(E, F_{3}\right) \leq 3.5 \tau_{n}\left(\left|x-e_{1}\right|\right)
$$

as in Case 2.

So the lemma is proved in all cases.

4.16. Lemma. If $x \in\left[e_{1} / 2, e_{1}\right] \backslash\left\{e_{1}\right\}, p(x) \leq 4 \tau_{n}\left(\left|x-e_{1}\right|\right)$.

Proof. We apply the same map $T$ as in the proof of Lemma 4.13. Then $T(x)$ lies on the unit circle; (we continue to identify the $e_{1} e_{n}$-plane with $\mathbb{C}$ as in the proof of Lemma 4.13). Let $T(x)=y=y_{1}+i y_{n}$ and $S=S^{n-1}\left(y, y_{n}\right)$. Consider the following sets: $K_{1}=\left[y,\left(1+y_{n}\right) y\right], K_{2}=\left[\left(1+y_{n}\right) y, \infty\right], K_{3}=$ $\left[-e_{n},-3 e_{n}\right] \cup\left[-3 e_{n}, \infty\right]$, and $E=\left[-e_{1}, e_{1}\right]$. Then

$$
p(x) \leq \sum_{j=1}^{3} M\left(E, K_{j}\right) .
$$

By Lemma 4.3,

$$
M\left(E, K_{3}\right) \leq 2 \tau_{n}\left(\frac{1}{2}\right) \leq 2 \tau_{n}\left(\left|x-e_{1}\right|\right) .
$$

Also, since $K_{2}$ is the reflection in $S$ of $K_{1}$, a polarization argument shows that

$$
M\left(E, K_{1}\right) \leq M\left(E, K_{2}\right) .
$$

To estimate $M\left(E, K_{2}\right)$, we apply Theorem 1.4 to get

$$
M\left(E, K_{2}\right) \leq \tau_{n}\left(\frac{y_{n}}{2}\right) .
$$

We compute $y_{n}$ in terms of $x=x_{1} e_{1}$ :

$$
T(x)=\frac{1-\left(2 x_{1}-1\right) i}{2 x_{1}-1-i}=\frac{2 x_{1}-1+2 x_{1}\left(1-x_{1}\right) i}{2 x_{1}^{2}-2 x_{1}+1} .
$$

Therefore

$$
\tau_{n}\left(\frac{y_{n}}{2}\right)=\tau_{n}\left(\frac{x_{1}\left(1-x_{1}\right)}{2 x_{1}^{2}-2 x_{1}+1}\right) .
$$


But $x_{1} \geq 2 x_{1}^{2}-2 x_{1}+1$ for all $x_{1} \in[1 / 2,1]$ ! Hence (4.21) and the monotonicity of $\tau_{n}$ imply

$$
\tau_{n}\left(\frac{y_{n}}{2}\right) \leq \tau_{n}\left(1-x_{1}\right)=\tau_{n}\left(\left|x-e_{1}\right|\right)
$$

Now, (4.17), (4.18), (4.19), (4.20), and (4.22) yield

$$
p(x) \leq 2 \tau_{n}\left(\left|x-e_{1}\right|\right)+2 \tau_{n}\left(\left|x-e_{1}\right|\right)=4 \tau_{n}\left(\left|x-e_{1}\right|\right)
$$

and the lemma is proved.

Acknowledgements: I express my gratitude to Matti Vuorinen who suggested the problems to me and gave me many ideas and suggestions. He also read earlier versions of the paper and made several corrections.

The research in this paper has been carried out during my visit in the University of Helsinki with a grant from the Finish Ministry of Education (CIMO).

\section{References}

[AVV] Anderson G.D., Vamanamurthy M.K., and Vuorinen M., Conformal Invariants, Inequalities, and Quasiconformal Maps, Wiley, New York, 1997.

[BBH] Barth K.F., Brannan D.A., and Hayman W.K., Research problems in complex analysis, Bull. London Math. Soc. 16 (1984), 490-517.

[Du] Dubinin V.N., Transformation of condensers in space, Soviet Math. Dokl. 36 (1988), 217-219.

[G1] Gehring F.W., Rings and quasiconformal mappings in space, Trans. Amer. Math. Soc., 103 (1962), 353-393.

[G2] Gehring F.W., Extremal length definitions for the conformal capacity of rings in space, Michigan Math. J. 9 (1962), 137-150.

[Ku] Kuz'mina G.V., Moduli of Families of Curves and Quadratic Differentials, Proc. Steklov Inst. Math., 1982, issue 1. (Russian original: Tom 139, 1980).

[SV] Solynin A.Yu., Vuorinen M., Extremal problems and symmetrization for plane ring domains, Trans. Amer. Math. Soc. 348 (1996), 40954112.

[Te] Teichmüller O., Untersuchungen über konforme und quasikonforme Abbildung, Deutsche Math. 3 (1938), 621-678.

[V1] Vuorinen M., Conformal Geometry and Quasiregular Mappings, Lecture Notes in Math. 1319 (1988), Springer-Verlag, Berlin-New York. 
[V2] Vuorinen M., Conformally invariant extremal problems and quasiconformal maps, Quart. J. Math. Oxford Ser. (2), 43 (1992), 501-514.

[Wo] Wolontis V., Properties of conformal invariants, Amer. J. Math. 74 (1952), 578-606.

[Zi] Ziemer W.P., Extremal length and conformal capacity, Trans. Amer. Math. Soc., 126, (1967), 460-473. 